\title{
Geodynamic modeling
}

\author{
Huilin Xing · Jianhua Wang
}

Published online: 14 June 2009

(c) Springer-Verlag 2009

Idealized experiments and field observations have been the main pillars in geosciences and geoengineering for decades, while the rapid development of supercomputers leads to a paradigm shift towards numerical simulation and modeling of problems in these areas. Simulations based on high performance computing offer outstanding opportunities to get insights into increasingly complex geodynamic problems.

This special issue contains contributions to International Workshop on Advances in Computational Geomechanics and Geophysics (IWACGG), which was held in conjunction with the International Conference on Computational Science (ICCS) in Beijing, China, May 27-30, 2007. IWACGG received 82 contributions, and six of them were selected to be published in this special issue focusing on geodynamic modeling.

The contributions highlight recent research in geodynamic modeling, covering advanced computational techniques (including the finite element, boundary element, finite difference, and discrete element methods), geomaterials database, high performance software development on supercomputers and their applications spanning different temporal and spatial scales, such as earthquakes, tsunami, subduction process, rock physics and the entire earth behaviors.

We would like to thank Professor Wu and Professor Borja for providing this opportunity to publish selected scientific contributions in Acta Geotechnica.

H. Xing $(\bowtie)$

Earth System Science Computational Centre,

The University of Queensland,

Sir James Foots Building (47a),

St Lucia, QLD 4072, Australia

e-mail: xing@esscc.uq.edu.au

J. Wang

School of Naval Architecture,

Ocean and Civil Engineering,

Shanghai Jiao Tong University,

1954 Huanshan Road, 200030 Shanghai, China 